\title{
HIPÓTESIS, CITAS, RESULTADOS: REFLEXIONES SOBRE LA COMUNICACIÓN CIENTÍFICA EN DIDÁCTICA DE CIENCIAS
}

\author{
JIMENEZ ALEIXANDRE, M.P. y GARCÍA-RODEJA GAYOSO, I. \\ Departamento de Didáctica das Ciencias Experimentais. Universidade de Santiago de Compostela. \\ Escola de Maxisterio. Av, Xoán XXIII, s/n. 15704 Santiago de Compostela.
}

\section{SUMMARY}

This paper raises a reflection on the role of scholarly papers in the dissemination of research in Science Education, and in the discussion about educational theories. The convenience of a rigorous distinction in each paper between what are hypotheses or opinions, what are evidences, experimentally tested, and what constitutes a metanalysis from literature review, is suggested. These suggestions attempt to improve the quality of scientific communication in the community doing research in Science Education.

\section{INTRODUCCIÓN: LA COMUNICACIÓN CIENTÍFICA EN DIDACTICA DE CIENCIAS}

El papel de una revista académica es el de servir de vehículo de comunicación entre la comunidad científica y profesional de un campo determinado. Mediante las revistas, las personas que investigan en un dominio pueden conocer el trabajo realizado por otros equipos y dar a conocer el suyo; los profesionales que trabajan en ese campo -en el caso de la educación, el profesoradopueden informarse acerca de los resultados de los estudios, y ambos grupos, investigadores y docentes, pueden compartir reflexiones sobre los problemas del campo.

A su vez las revistas no son meros espejos que reflejan lo que sucede en la investigación, sino que juegan un importante papel en la delimitación de los problemas a estudiar, de los métodos de estudio, de los modelos aceptados o marginados en un momento dado $y$, por supuesto, en el establecimiento de unas pautas más o menos convencionales sobre cómo comunicar los resul tados de la investigación. En otras palabras, las revistas tienen un papel formal, el de canal de comunicación, y otro, quizá menos explícito pero ampliamente reconocido, como fuerza o poder que opera en la comunidad acadómica y profesional. Algunos ejemplos de este rol pueden ser la resistencia de las revistas educativas a aceptar estudios de carácter cualitativo a comienzos de los ochenta (Shuiman, 1996), la difusion de las ideas de Ausubel a través de Science Education en la misma época, en oposición al Journal of Research in Science Teaching de orientación más piagetiana, o el papel de Enseñanza de las Ciencias en la difusión de las ideas constructivistas en España.

La comunidad a la que se refiere este artículo es ia de didáctica de ciencias. Es un campo relativamente nuevo y una comunidad emergente (en palabras de Aliberas, Gutiérrez e Izquierdo, 1989), en la que los problemas están aún đefiniéndose y no hay un consenso sobre los paradigmas teóricos. Prueba de su juventud es que sólo una de las revistas fundamentales del campo, Science Education, tiene una antigüedad que se remonta a 1916, mientras que otras que en la actualidad cuentan con gran prestigio, como el Journal of Research in Science Teaching y el International Journal of Science Education, datan respectivamente de 1963 y 1979 . Recientemente se estásuscitando un debate en el seno de algunas de estas revistas acerca de su propio papel 
(Duschl, 1994; Gilbert, 1994), debate que quizá esté relacionado con esta juventud por un lado y, por otro, con las transformaciones y discusiones relacionadas con los procesos de reforma emprendidos en distintos países. Entre las publicaciones en castellano, Enseñanza de las Ciencias ha desempeñado desde su aparición en 1983, un papel fundamental en la vertebración de la comunidad de didáctica de ciencias, no sólo en España, sino también poniéndonos en comunicación con Latinoamérica y con otros países mediterráneos. Esta revista se dirige de forma explícita a los dos sectores -investigadores y docentes- a que antes nos referíamos, por lo que incluye tanto trabajos que detallan resultados de investigaciones, como otros centrados en la enseñanza.

El objetivo del presente trabajo es realizar una reflexión crítica sobre el discurso que se realiza en los artículos, sobre los problemas estudiados y las bases metodológicas de los trabajos que se exponen en ellos, sobre los modelos epistemológicos implícitos y sobre la forma de presentar los resultados. La intención de las autoras es contribuir a mejorar la calidad de la comunicación científica sobre didáctica de ciencias, particularmente en la comunidad latina, señalando algunas -a su juicioinsuficiencias y proponiendo líneas de progreso. Más que criticar trabajos concretos, se proponen poner de manifiesto algunas deficiencias comunes a un número relativamente importante de éstos, y que, puesto que se aprende a escribir artículos leyendo otros, se van transmitiendo de unas revistas a otras, de unos números a otros. Esta reflexión se refiere no sólo a los trabajos publicados en Enseñanza de las Ciencias, sino a los aparecidos en otras revistas, así como a los presentados en congresos. Sería deseable que se abriera un debate sobre estas cuestiones, ya que es probable que haya opiniones y propuestas diferentes de las que se exponen aquí. En otras palabras, ćsta pretende ser una reflexión constructiva, que permita avanzar en el camino de estructurar la comuniđad latina en didáctica de ciencias, comenzado por Enseñanza de las Ciencias, aumentando el nivel de autoexigencia y proporcionando algunas pautas críticas de análisis. Otros artículos han tratado de estos primeros diez años de la revista (Gil, 1994; Moreira, 1994), analizando la naturaleza de los artículos publicados y las líneas investigadas.

A continuación se discute, en primer lugar, la relación entre comunicación e investigación, comenzando por el tipo de problemas investigados; en el tercer apartado se aborda el papel de la revisión de la literatura en la investigación y la comunicación científica, y la forma en que esto se refleja en el discurso de los artículos; en tercer lugar se abordan algunos de los modelos epistemológicos y las bases metodológicas explícitos -o en muchas ocasiones implícitos- en los trabajos; por último se discute la presentación de resultados y las conclusiones que de ellos se derivan.

\section{RELACIÓN ENTRE ARTÍCULOS E INVESTIGACIONES: PROBLEMAS INVESTIGADOS}

Discutir la relación entre investigaciones y artículos puede parecer una obviedad, pero teniendo en cuenta que, por una parte, pocas veces se trata explícitamente esta relación y, por otra, que hay artículos que no dan cuenta de una investigación, sino que abordan problemas teóricos o metodológicos -como éste, por ejemplo-, cabe dedicar algunas líneas a precisarla. Una cuestión que parece evidente y no lo es tanto (a juzgar por algunas confusiones) es la existencia de diferentes tipos de artículos. Por ejemplo, en una revisión bibliográfica sobre educación ambiental (López et al., 1992) hemos agrupado los trabajos en seis categorías, que presentamos con algunas modificaciones en la tabla $X$.

\begin{tabular}{|c|c|}
\hline CATEGORÍAS DE TRABAJOS & EJEMPLOS \\
\hline 1. Reflexjones téricas o metodológicas & Fundamentación, debates metodotógicos, revisiones. \\
\hline 2. Investigaciones didácticas & Informes sobre investigaciones. \\
\hline 3. Experiencias & Proyectos y estudios realizados en el aula \\
\hline $\begin{array}{l}\text { Trabajos descriptivos (no recogen investigaciones } \\
\text { ni experiencias) }\end{array}$ & \\
\hline $\begin{array}{l}\text { 4. Propuestas de experiencias } \\
\text { 5. Descripción de técnicas y recursos } \\
\text { 6. Actualización discipinar }\end{array}$ & $\begin{array}{l}\text { - Propuestas no experimentadas } \\
\text { - Sin resultados de su utilización } \\
\text { - Puesta al día o debatcs disciplinares }\end{array}$ \\
\hline
\end{tabular}


En su análisis sobre Enseñanza de las Ciencias, Moreira (1994) establece seis categorías, de los que «investigaciones" (junto con «análisis de textos») se corresponderían con el 2 presentado aquí, «revisión y reflexión» (junto con «epistemología») con el 1 , «métodos» con el $3, y$ «descripciones» con 4,5 y 6 .

Por ejemplo, muchos de los trabajos aparecidos en Studies in Science Education pertenecen a la primera categoría (reflexiones teóricas, debates metodologicos, revisiones), como el de Solomon (1994); en cuanto a artículos publicados en Enseñanza de las Ciencias, el de Aliberas y otros (1989) pertenece a ellos. Son trabajos muy interesantes y su existencia es necesaria en las revistas a condición de que posean ciertas características, como: proponer ideas novedosas, recoger de forma rigurosa y documentada las ideas y debates existentes sobre un tema, tener en cuenta la literatura anterior, sistematizar de aigún modo los trabajos o las diferentes posturas sobre una cuestión, etc. En otras palabras, en muchos casos resulta más difícil elaborar un buen artículo de reflexión o revisión que uno que recoja una investigación, ya que no se trata de lanzar especulaciones o de proponer opiniones sin fundamento, sino de articular ideas de forma que resuiten más útiles; si realizar buenas investigaciones no es fácil, aún lo es menos tener ideas originales desde el punto de vista teórico, y la historia de la ciencia parece indicar que las grandes ideas teóricas surgen de un profundo conocimiento de las ideas precedentes.

En cuanto a la segunda categoría -investigaciones didácticas-, constituye la mayor proporción de los artícuIos en las revistas científicas, y las que hemos citado de didáctica de ciencias no son una excepción; en Enseñan. za de las Ciencias, según Moreira (1994), representan el $43 \%$. A continuación nos referiremos específicamente a los artículos que dan cuenta de investigaciones.

En un artículo que pretende hacer explícita la línea de la revista, Richard DuschI (1994), director de Science Education, plantea los criterios que guiarán la selección de trabajos para su publicación. Aunque se trata de 10 que será aceptable o no en los trabajos escritos, todos los temas tocados (líneas de investigación, selección de problemas, discño...) son relevantes para la investigación. En otras palabras, al definir los criterios para artículos de calidad, Duschl está también señałando pautas de lo que -en su opinión- constituye investigación de calidad. Existe una estrecha relación entre ambas cosas, pues, como se ha indicado en un comentario sobre este artículo (Jiménez, 1995), de una buena investigación pueden salir buenos artículos, o también a veces malos artículos, pero de una investigación con deficiencias es difícil que se origine un buen artículo.

El primer elemento esencial para una buena investigación y un buen artículo es que aborde un problema interesante; por usar los términos de Bachelard (1938), que plantee una buena pregunta. Refiriéndose a los problemas investigados, DuschI señala que son necesarios más estudios que versen sobre problemas relevantes, ya que, por ejempio, existen pocos estudios que examinen en el aula cómo impartir la instrucción que proponen los programas relacionados con reformas (cursivas de Duschl); por el contrario, indica como ejemplo de elección poco adecuada informes endebles sobre errores conceptuales o ideas alternativas que denomina «a favor de la corriente» (bandwagon). El problema no es que la cuestión sea poco interesante, o que existan ya muchos estudios sobre ella, sino que estos trabajos son endebles porque no desarrollan conexiones con investigaciones anteriores ni realizan contribuciones claras acerca de qué dirección tomar en el futuro o qué preguntas plantear (Duschl, 1994, p. 206).

La cuestión de tos problemas a investigar ha sido abordada también por Lucas ( 1990 ), quien realiza un toque de atención sobre las cuestiones que pueden estar de moda en cada momento, y apunta a la necesidad de conexión entre la investigación y la práctica del aula, poniendo como ejemplo el proyecto PEEL (Baird y Northfield, 1992). En el análisis realizado por Moreira (1994) sobre los diez primeros años de Enseñanza de las Ciencias, se indica que el $38 \%$ de los artículos de investigación versan sobre las ideas alternativas y el cambio conceptual.

La definición del problema es muy importante. Estudios sobre problemas poco definidos pueden cumplir un papel de iniciación a la investigación, a igual que los que replican trabajos realizados en otros lugares, pero en el futuro, si se pretende realizar aportaciones a la investigación en didáctica de ciencias, habrá que esforzarse en buscar problemas más originales. Nos atrevemos a sugerir algunas ideas:

- El análisis decallado de lo que sucede en las clases de ciencias en las cuales se llevan a cabo proyectos innovadores -creemos que la proporción de estas clases en España es muy alta, en comparación con otros países de nuestro entorno- puede ser una línea que arroje luz sobre aspectos aún oscuros de la forma en que se aprenden las ciencias.

- Es importante estudiar los procesos y problemas de aprendizaje analizando secuencias completas de trabajo en el aula (por ejemplo, mediante análisis del discurso), y no sólo productos mediante calas puntuales (pasar una o varias pruebas en momentos escogidos).

- Necesitamos más estudios longitudinales que nos indiquen qué parte de lo aprendido se recuerda, se usa, se transfiere a otros contextos.

- Además de lo que las y los estudiantes saben de contenidos de ciencias, o piensan sobre lo que es la ciencia, interesa saber cómo hacen las cosas que hacen (experimentar, proponer hipótesis, comparar teorias, razonar, argumentar) en un contexto de indagación.

Otro aspecto al que debe prestarse más atención es la conexión con investigaciones anteriores: en trabajos que pretenden ser rigurosos, es poco admisible que se ignoren estudios sobre los mismos problemas, que se planteen cumo si se partiera de cero. 
El siguiente apartado hace referencia con más detalle a la revisión de la literatura, pero conviene aclarar que, si un trabajo pretende insertarse en una línea de investigacion, debe establecer de forma clara tanto el punto de partida, es decir, lo que han avanzado otros estudios anteriores y en qué medida es deudor de ellos, como la dirección de progreso, o sea, lo que aporta de nuevo a la línea y los caminos que abre para futuros estudios.

En cuanto al formato, tradicionalmente los trabajos de investigacion se presentan con estos apartados: a) planteamiento del problema, definiendo su contexto, y revisión de antecedentes (lo que otros han hecho sobre el mismo); b) metodología, incluyendo hipótesis a contrastar, muestra, instrumentos de recogida de datos y método de análisis; c) resultados, presentados por medio de tablas y en el texto; y $d$ ) discusion e implicaciones, en el caso de la didáctica, para la enseñanza. Aunque este esquema puede parecer un poco rígido y en ocasiones un trabajo se pueda salir de él, en general facilita la comprensión por parte de las personas que lo leen y la comparacion con otros estudios. Sin embargo, hay trabajos publicados en Enseñanza de las Ciencias, en otras revistas y más a menudo en actas de congresos, donde no hay selección, o es menos estricta, en los que es difícil saber, por ejemplo, qué problema concreto ha dado Iugar al estudio y qué hipótesis se desea contrastar, o en los que no se especifica claramente la muestra estudiada, o en los que se ignoran las implicaciones del estudio para las clases de ciencias. Así pues, la llamada de atencion que hacemos no se dirige sólo a las autoras y autores, sino también al consejo asesor y a la direccion de la revista, responsables de la calidad general de los artículos.

En las revistas académicas caben también otro tipo de trabajos, como los que describen experiencias y proyectos o incluso los que realizan propuestas no experimentadas, y que en nuestra clasificación corresponden a las categorías 3 al 6 . Sin embargo, en nuestra opinión, la mayor proporción de los trabajos debería corresponder a las dos primeras categorías de la tabla I, especialmente a las investigaciones, y los de carácter descriptivo deberían apoyarse en unas sólidas bases teóricas, así como en una revisión de la literatura anterior, para no presentar como novedosas propuestas que ya han sido publicadas; o en todo caso, se tendría que precisar lo que contienen de nuevo.

\section{EL PAPEL DE LA REVISIÓN BIBLIOGRAFICA Y SU REFLEJO EN EL DISCURSO}

Una vez definido claramente el problema a investigar, el siguiente paso es la revisión de la literatura existente sobre una cuestión determinada. El objetivo de esta revisión es, por una parte, situar el problema estudiado en un marco teorico determinado, en el conjunto de cuestiones y problemas sobre los que una comunidad trabaja en un momento dado; por ejemplo, Black y Lucas (1993) indican que muchos trabajos sobre ideas de los estudiantes no se enmarcan en el contex to de una teoría de aprendizaje. Por otra parte, también tiene como objetivo conocer lo que se ha progresado en un campo determinado para avanzar a partir de ello; es decir, "apoyarse en hombros de gigantes» y contrastar los resultados propios con los de otros estudios.

La calidad de esta revisión en los artículos que dan cuenta de las investigaciones, o en las reflexiones teóricas, es uno de los criterios que tradicionalmente utilizan las revistas para evaluar la calidad de los trabajos. Esto, que en principio es un criterio válido, puede dar lugar a ciertos hábitos de acumular citas poco relevantes, de referirse a trabajos sin haber entendido realmente la esencia de su discurso, o incluso de citar autores sin que el texto muestre que se conoce su trabajo, todo lo cual repercute en el rigor de los artículos.

La función de una referencia es, por ejemplo, indicar en qué fuentes teoricas y empíricas se basa el autor o autora. La coherencia con posiciones epistemologicas como las de la nueva filosofía de la ciencia, siguiendo la nomenclatura de Estany (1993), que critican no sólo el empirismo sino las posiciones filosoficas tradicionales, parece que debería llevar a la mayoría de los profesionales del campo a citar no por el principio de autoridad sino porque se esté de acuerdo -o no se esté de acuerdo-con un trabajo determinado. En otras palabras, desde estas posiciones epistemológicas no se puede dar por buena tal o cual opinión o teoría porque provenga de una autoridad determinada, sea Ausubel, Piaget o Popper, por muy prestigiosa que sea. Con mayor motivo, las citas de un trabajo deberían corresponder preferentemente al aspecto esencial tratado en el mismo, recoger lo substancial, no frases fuera de contexto; por ejemplo, si una persona se ha dedicado fundamentalmente a investigar cuestiones relacionadas con la demanda cognitiva, y sus artículos tratan sobre eso, no sería muy riguroso emplear una frase de un artículo suyo para apoyar tal o cual posición sobre los trabajos de laboratorio.

En la misma línea, es preciso distinguir claramente, cuando se menciona una referencia, si corresponde a una investigación o a una reflexión. La razón es que, mientras que en el primer caso podría decirse que $N$ ha mostrado..., o los trabajos de $N$ indican que..., en el segundo caso, lo adecuado sería escribir que $N$ opina que... o según piensa $N$... De un artículo de reflexión o de la discusión đe resultados de un trabajo, no puede deducirse que se ha demostrado alguna cosa -como se trata más adelante, tambiên es discutible lo que «muestran» los propios resultados-, sino únicamente la opinión, por muy fundamentada que sea, de la autora o autor de 1 trabajo.

Al citar a otra persona para apoyar una frase, debe quedar lo más claro posible qué parte de la frase se apoya. No sería muy ilustrativo escribir las ideas de los estudiantes (Driver, Viennot, Novak)..., porque, en primer lugar, un concepto como «las ideas de los estudiantes» no es una aserción teorica ni metodologica que requiera un apoyo en referencias $y$, por otra, porque los diferentes autores que se citan en un mismo paréntesis pueden tener -y de hecho tienen en muchas ocasiones- posiciones diferen- 
tes acerca de «las ideas de los estudiantes». Hacer referencia a varios autores juntos es adecuado sólo en algunas ocasiones: si se mencionan varios estudios sobre un tema sin hacer hincapié en las diferencias, $E n$. distintos paises se han estudiado las dificultades de aprendizaje de la fotosíntesis (Barker y Carr, Stavy, Wandersee); si se citan trabajos de una misma categoría sobre temas distintos, Revisiones bibliográficas (Cervantes, Furió, Serrano); si se citan varios irabajos de un mismo equipo, Los trabajos sobre destrezas cognitivas de Kuhn y colaboradores (Kuhn 199-, Kuhn, Schauble y García-Milá); y en otros casos pareciodos. Sería una simplificación excesiva, por el contrario, reunir varias citas en un mismo paréntesis para ilustrar una posición teórica o metodológica, ya que éstas raramente coinciden por completo, y es más riguroso indicar en qué aspectos coinciden y en qué matices difieren.

Otra cuestión diferente es la de citar artículos sin comprender lo substancial de su discurso. Esto suele ocurrir sobre todo con autoras o autores muy citados, a los que gran número de personas que estudian o escriben sobre un tema se sienten en la obligación de mencionar, pero que conocen sólo superficjalmente. Tomando como ejemplo el «cambio conceptual», tanto el artículo de Posner, Strike, Hewson y Gertzog (1982) -mencionado a veces como PSHG-como el de Hewson (1981) se encuentran entre los más citados en la última década, y el «cambio conceptual» es una idea manejada a menudo. Sin embargo, en ocasiones, la forma en que se emplea el ucambio conceptual» es una versión distorsionada, o que contradice abiertamente las ideas contenidas en estos artículos. Por ejemplo, como se discute en Jiménez (1991), el artículo de Hewson (1981) tiene como objetivo explícito ampliar el modelo de «cambio conceptual» al caso en que las ideas previa y nueva no son incompatibles -modalidad que denomina «captura»-y diferenciarto de la modalidad «intercambio" que sería el caso en que dichas ideas fueran irreconciliables. No tiene sentido por tanto, definir el «cambio conceptual» como la sustitución de una idea por otra incompatible con la primera, citando a Hewson; o tiene sentido definirla así si se hace constar que ésta es la opinión del autor, y por qué no está de acuerdo con Hewson. Otra distorsión del modelo propuesto por Posner y otros (1982) consiste en referirse a las condiciones para el «cambio conceptual» (insatisfacción con la idea previa; inteligilibilidad, plausibilidad y fertilidad de la idea nueva) como si fuera el docente quien ha de juzgar en qué momento se cumplen, ya que según el modelo es el propio estudiante quien debe decidir cuándo una idea es o no satisfactoria, comprensible o creíble para ella oél (Hewson y Thorley, 1989). En consecuencia, es preferible citar menos trabajos, pero que se conozcan en profundidad. Una cita no constituye una invocación, sino que debe ser una herramienta de trabajo; un artículo, algo que usamos para extraer ideas, modificarlas, usar sus métodos o criticarlos, en definitiva, una porción más de nuestro propio trabajo.

En este ejemplo nos hemos referido a la cita de trabajos con los que se está en desacuerdo. De hecho, una revisión bibliográfica no tiene por qué incluir solamente a los autores con los que se concuerda, al contrario, en una revisión exhaustiva habrá trabajos con los que se esté más de acuerdo, otros con los que se esté en desacuerdo, y otros con los que se esté de acuerdo sólo parcialmente. Cuanto más se trabaje una cuestión y más se profundice en ella, en mayor medida se irá construyendo un discurso propio que coincidirá en parte con aportaciones anteriores, pero que ampliará o incluso contradirá ciertos aspectos de trabajos previos. Las revistas han albergado numerosas polémicas y, de hecho, ése es el objetivo de la sección de comentarios y críticas (que, por cierto, pocas veces se utiliza en Enseñanza de las Ciencias para este fin).

De lo dicho anteriormente se deduce que, en nuestra opinión, la revisión es muy importante. Cabe insistir en que, aunque la revisión tenga fallos, es mejor una revi* sión incompleta que la ausencia de ella y que debería ser menos frecuente cada vez encontrar artículos de autores que apenas citan otros trabajos o que sólo se citan a sí mismos. El conocimiento no surge de la nada, se apoya en otro conocimiento anterior, annque sea para rechazarlo.

\section{IDEAS, HIPÓTESIS: MODELOS EPISTEMOLÓGICOS Y BASES METODOLOGICAS}

Una preocupacion constante en las investigaciones en didáctica de las ciencias -y en general en educación-es la fundamentación teórica de estos trabajos. Parte de esta fundamentación se toma de otros campos, especialmente de la filosofj́a de la ciencia y de la psicología del aprendizaje, ya que se considera por parte de muchas personas que estas dos materias y la didáctica de las ciencias comparten problemas comunes y se interesan en el desarrollo del conocimiento científico; es decir, en términos de Shapere, las tres constituyen un «dominio» (Duschl y Hamilton, 1992). Algunas ideas y herramientas metodológicas se han tomado también de la linguifstica (análisis del discurso), de la antropología (métodos etnográficos) o de la sociología. Debido tal vez a las críticas al empirismo realizadas en el campo educativo en Ias últimas décadas, las autoras y autores se preocupan por no ser «empiristas», por mostrar que trabajan dentro de un marco teórico, dentro de un programa coherente. Esta preocupación es laudable, pero quizá puede explicar que, en muchos trabajos, los autores se sientan obligados a comenzar por un apartado de fundamentacion epistemológica que a veces ofrece una versión simplificadora, cuando no distorsionada, a ojos, por ejemplo, de una persona especialista en filosofía de la ciencia.

En este trabajo no cabe un análisis detallado de diferentes concepciones históricas, que puede encontrarse en Estany (1993) o, en cuanto al paralelismo entre ellas y los modelos de enseñanza, en Mellado y Carracedo (1993). Sí interesa destacar algunos aspectos; en primer lugar, que además de las críticas al empirismo también cabe criticar la filosofía de la ciencia tradicional, puesto 
que algunos de sus aspectos -el principio de autoridad mencionado más artiba, que basa la veracidad de un modelo o teoría en la autoridad de quien lo sostieneticnen influencia en la enseñanza (Russell 1983). En segundo lugar, hay que reconocer que el empirismo, como corriente fjlosófica, ha sido superado hace tiempo, aunque no ocurra to mismo con su influencia en la enseñanza. En tercer lugar, no parece riguroso hablar de fillosofía de la ciencia «actual», o «correcta», o «apropiada" como alternativa al empirismo, ya que, por un lado, existen diferentes corrientes filosóficas actuales y, por otro, hablar de una posición «correcta» es contradictorio con esas nuevas corrientes -llamémoslas nueva filosofía de la ciencia, o historicistas, que son algunos nombres que reciben en los manuales de filosofía-. Según estas corrientes, distintos observadores perciben las mismas cosas de formas diferentes, por lo que no cabría hablar de posición correcta, sino sólo de correcta para mi, o correcta para $N$, siendo $\mathrm{N}$ un autor determinado. En otras palabras, quizá sea más adecuado dar a cada posición filosofica un nombre, preferiblemente el más cmpleado en el propio campo de la filosofía de la ciencia, o en todo caso asignarla al autor que la defiende. A veces también se habla de posiciones constructivistas (en filosofía de la ciencia), y también cabría matizar que éstas no son homogéneas.

Siguiendo a Estany (1993) puede distinguirse, por un tado, la llamada nueva filosofía de la ciencia, representada por autores como Kubn, Lakatos o Toulmin y, por otro, dentro de los enfoques teórico-modelistas, la concepción estructural, con autores como Moulines, y la concepción semántica, siendo dentro de ésta un autor de gran influencia Ronald Giere (1988), quien propone un enfoque cognitivo, en el sentido de utilizar los conceptos y métodos de las ciencias cognitivas para estudiar la ciencia. Esto se traduce en considerar la ciencia como una actividad cognitiva, relacionada con la generación de conocimientos, en la que el proceso de decidir entre diferentes modelos se contempla como un ejemplo del cjercicio humano de juzgar, más gue como un proceso de inferencia. Otros autores de filosofía e historia de la ciencia situados en la perspectiva cognitiva son William Bechtel, quien analiza casos de la biología moderna, o Paul Thagard (1992), que utiliza modelos de computación para analizar la coherencia explicativa de distintas teortas.

Este resumen tan esquemático sólo pretende poner en evidencia la complejidad del campo y reclamar mayor cautela a la hora de situarnos en un marco epistemológico, y sobre todo de defínirlo o calificarlo, sugiriendo en todo caso citar las fuentes de las que se ha tomado tal definición o clasificación. No podemos convertirnos en especialistas en filosofía o psicología, pero podemos estar atentos a lo que estos campos nos pueden ofrecer.

En algunos trabajos, por ejemplo, sobre modelos de aprendizaje, las críticas a otras posiciones revisten sobre todo un carácter de descalificaciones; se califica un modelo de «inadecuado», «incorrecto», «superficial» $\mathfrak{u}$ otros adjetivos con carga peyorativa sin explicar detalla- damente por qué se opina así. Por supuesto que en muchas ocasiones opinaremos que un modelo o un método son «inadecuados», pero será por alguna razón. En la dirección de precisar mejor las distintas posiciones sería deseable que, si un autor propone un modelo, cite no sólo las fuentes con las que está de acuerdo, sino que analice los modelos existentes, los critique en profundidad explicando por qué no está de acuerdo con ellos y señale en qué aspectos el nuevo modelo propuesto mejora los existentes. Así hicieron, por ejemplo, los psicólogos partidarios de las teorías cognitivas, criticando el conductismo; o así hace Novak (1982) en su libro sobre el aprendizaje significativo. En su versión más reciente, el manual de estilo de la Asociación Americana de Psicología (APA, 1994), al que deben ajustarse los artículos para la mayoría de las revistas de habla inglesa en nuestro campo, recomienda evitar las calificaciones y valoraciones. Según Osborne y Wittrock (1985), un modelo sobre aprendizaje es una simplificacion de la realidad, y hay que tener en cuenta que distintos modelos pueden ser útiles para distintos propósitos.

En cuanto a los aspectos metodologicos, es imposible referirse a todos eltos de manera detallada. También aquí cabría recomendar la consulta bibliográfica, pues existe un gran numero de manuales para asegurarse de que el diseño es lo más riguroso posible. Ya hemos mencionado que el planteamiento del problema es un aspecto esencial, y hay que asegurarse de que existe una correspondencia entre el problema investigado y los métodos empleados. Quizá el conocimiento de los múltiples problemas que presenta el aprendizaje de las ciencias ha llevado a querer abarcar demasiado en algunos estudios: diagnosticar, tratar y curar todo de una vez. Sin embargo, parece que los estudios más profundos requieren un campo de estudio más delimitado, y la recomendación sería de nuevo prudencia. Es posible también optar por un problema menos localizado, pero más contextualizado en el trabajo del aula, más relevante para las clases de ciencias de cada día; en ese caso es importante ser conscientes de que el trabajo será menos generalizable -lo cual no quicre decir menos valioso-y que así habrá de reflejarse en las conclusiones.

En cuanto a la toma de muestras, es importante especificar por qué se elige esa muestra, y qué características tiene. Como indica Duschl (1994), no es lo mismo estudiar una muestra accidental (centros o cursos a los que se tiene fácil acceso por motivos personales o profesionales) que una escogida al azar y representativa. Una cuestión relacionada con las muestras es la diferencia entre estudios cuantitativos y cualitativos -y por supuesto que las muestras a estudiar serán muy diferentes en unos y otros-. Como hemos discutido en otro lugás (Jiménez, 1992), ambas metodologías son complementarias, no opuestas; es decir, que pueden proporcionar imágenes de diferentes aspectos de un problema. Pero ello no significa que la metodología cuatitativa no tenga sus propias normas y métodos; podemos citar los manuales de Burgess (1985), de Sherman y Webb (1988) o el de Blanchet y otros (1989) traducido al castellano. Por ejemplo, los estudios de aula presentan bastantes dificultades y complicaciones y hay un gran volumen de 
trabajos que han intentado abordar cómo analizar rigurosamente el discurso en el aula (Hewson et al., 1992; Kelly y Crawford, 1996; Russell, 1983; Smith et al., 1992; Thorley, 1992); en otras palabras, son estudios de un tipo distinto de los cuantitativos, pero que conllevan un análisis laborioso, por lo que no se pueden etiquetar como «metodología cualitativa» unas meras impresiones sobre to que sucede en el aula de las que no se describe ni la forma en que se han tomado los datos, $\mathrm{ni}$ el procedimiento utilizado para analizarlos.

En cuanto a la presentación de resultados, tiene mucho que ver con la metodología, pues dependiendo de si son fundamentalmente cuantitativos o cualitativos será conveniente un tratamiento estadístico o no. La tendencia hoy día en muchos trabajos, al revés de lo que ha sucedido en otros momentos, es no considerar indispen. sable un fuerte tratamiento estadístico, pero naturalmente esto tiene relación con el problema estudiado, las hipotesis y las implicaciones. Si se pretende obtener una panorámica de lo que sucede en un país, en un dominio concreto, generalizar u obtener conclusiones que vayan más allá de un estudio de caso, es conveniente contar con muestras que cumplan ciertos requisitos y someterlas a un tratamiento estadístico. Incluso cuando se realiza un estudio de caso, es fundamental, al presentar los datos, describir con detalle en qué circunstancias se han tomado, por si alguna de ellas pudiera afectar a las conchusiones del estudio; un ejemplo puede ser: si la persona que investiga y la que imparte la instrucción son la misma y se está investigando precisamente el efecto de la instruc. cion, esta circunstancia ha de reflejarse explícitamente, y sería deseable contrastar los datos con otros en que la instrucción fuese impartida por una persona diferente. Esto no implica que un profesor o profesora no pueda investigar lo que sucede en su propia aula -al contrario, la tradición de la investigación-acción ha mostrado que dichos estudios son may valiosos- sino que se tenga en cuenta, por una parte, que hay cuestiones diferentes a la valoración de la efectividad de una instrucción y, por otra, que hay que tomar todas las precauciones posibles para evitar sesgos.

\section{RESULTADOS Y CONCLUSIONES: LA COMPLEJIDAD DE LOS PROBLEMAS EDUCATIVOS}

La presentación de resultados y la inferencia de conclusiones no están ex entos de problemas. En ocasiones, a la hora de escribir un artículo sobre una investigación que nos ha llevado varios años, estamos tan familiarizados con los datos que nos parece obvio todo lo que se refiere a ellos, y juzgamos superfluo añadir información. Sin embargo, para una persona que se enfrenta a ese trabajo por primera vez, unos resultados sobre los que no se da suficiente información pueden resultar casi incomprensibles. A continuación citamos algunos ejemplos de problemas concretos que hemos encontrado, si bien no creemos conveniente citar los autores o trabajos que presentan estas deficiencias.
Habitualmente la presentación de los resultados va acompañada de tablas, en las que se presentan Ios datos resumidos y sistematizados, pero en las que no debe faltar información. Por ejemplo, debe figurar el tamaño de la muestra, $N$, pues los porcentajes de las distintas categorías u otros valores no tienen el mismo significado para una muestra grande o pequeña. Un excelente trabajo acerca de cómo construir y mejorar tablas es el de Wainer (1992), quien hace notar que, al ser el error estándar de cualquier prueba estadística proporcional a $1 / \sqrt{n}$, siendo $n$ el tamaño de la muestra, sería necesaria una muestra de $40.000\left(200^{2}\right)$ para justificar presentar una correlación como 0,25 , lo que implica un error estándar menor de 0,005 .

Una primera medida que suele mejorar la calidad del artículo es una clara separación entre resultados y conclusiones. Y ello porque, de unos mismos resultados, puede llegarse a conclusiones diferentes. No se debe olvidar al presentar los resultados que -en coherencia con la nueva filosofía de la ciencia- el investigador o investigadora está percibiéndolos desde un marco deter. minado, y los atribuirá a unas causas; pero otras personas podrían atribuirlos a causas distintas. Esto que, como señalan Lucas y García-Rodeja (1990), debe ser tenido en cuenta para las experiencias en clase, con mayor motivo si se trata de una investigación. Supongamos una comparación entre dos grupos; es simplista atribuir unos resultados mejores en el experimental a un método determinado de instrucción I si además hay otros facto. res en los que los dos grupos difieren claramente: por ejemplo, si el método I implica un período de instrucción mucho más largo que el método estándar S. Por lo menos hay que incluir en la discusión una referencia a que los resultados también podrían deberse a esta mayor duración o a otros factores, y por qué razones se cree que no es así. La necesidad de esta discusión es aún mayor si otros trabajos han dado resultados que difieren de los presentados, o que encuentran causas distintas para los mismos. De la misma forma, es importante señalar las limitaciones de la investigación, qué aspectos quedan pendientes y qué interrogantes quedan abiertos.

También cabe recordar que no es lo mismo escoger una opción en un test de elección múfitiple que dar una respuesta a una entrevista o a una pregunta abierta. En el segundo caso puede ser adecuado decir que «los participantes creen que», pero quizá no debería emplearse esta formulación para estudios en los que se ha pedido a los participantes que elijan una opción entre varias, siendo en este caso más adecuado decir «tal porcentaje ha escogido tal opción». Más aún, hay casos en que un bajo porcentaje de participantes que escogen una respuesta es descrito como un rechazo de esa opción, cuando puede deberse a que los participantes han encontrado opciones que se correspondían mejor con su posición, o bien no han entendido la redacción de la misma forma que los investigadores. Una separación clara entre la descripción de las opciones y la forma en que los autores las interpretan puede evitar este problema.

Otro aspecto al que hay que prestar atención es la correspondencia entre los resultados y las conclusiones, 
pues, aungue parezca evidente que las conclusiones de un trabajo deberían guardar conexión con los resultados obtenidos, hay ocasiones en que los autores utilizan el apartado de conclusiones para exponer sus creencias sobre la enseñanza, sin relación ciara con los resultados del estudio. Claro está que, a partir de los resultados obtenidos, por ejemplo, acerca de la persistencia de determinadas ideas o del interés de los niños y niñas hacia la ciencia, la persona que investiga puede sugerir que la introducción de determinadas dimensiones en el currículo, como la atención a las ideas previas, la coherencia con la nueva fillosofía de la ciencia o las relaciones entre ciencia, tecnología y sociedad, podrían aumentar ese interés, o que la formación del profesorado debería incluir las cuestiones relativas al interés hacia la ciencia; pero este carácter de sugerencia -y la ausencia de una relación causa-efecto demostrada-debe quedar explícito en el trabajo. A lo largo de la historia de la educación ha ocurrido más de una vez que hipótesis sobre cómo solucionar un problema que parecían contar con una solida fundamentacion han sido refutadas empíricamente. En nuestra opinión, esto guarda relación con la propia complejidad de los problemas educativos, para los que raramente hay soluciones únicas, desvinculadas del contexto. Creemos que la aceptación de esta complejidad y,

\section{REFERENCIAS BIBLIOGRÁFICAS}

AI.IBERAS, J., GUTIÉRREZ, R. e IZQUIERDO, M. (1989). La didáctica de les ciències: una empresa racional. Enseñanza de las Ciencias, 7(3), pp. 277-284.

APA (1994). Publication Manual. American Psychological Association. Hyattsuille MD.

BAIRD, J.R. y NORTHFIELD, J.R. (eds.) (1992). Learning from the PEFI experience. Clayton, Australia: Monash University.

BACHELARD, G. (1938). La Formation de l'Esprit Scientifique. París: Vrín.

BLACK, P. y LUCAS, A.M. (1993). Children's Informal ldeas in Science. Londres: Routledge.

BLANCHET, A., GHIGLIONER., MASSONAT, J., TROGNON, A. (1989). Técnicas de investigación en ciencias sociales. Datos, observación, entrevista, cuestionario. Madrid: Narcea.

BURGESS, R. (ed.) (1985). Strategies of Educational Research: Qualitative Methods. Londres: The FalmerPress.

DUSCHL, R.A. (1994). Editorial Policy Statement and Introduction. Science Education, 78(3), pp. 203-208.

DUSCHL, R.A. y HAMILTON, R.J. (eds.) (1992). Philosophy of Science, Cognitive Psychology and Educational Theory and Practice. Albany, State University of New York Press. por tanto, el reconocimiento de que lo que se puede avanzar son pequeños pasos y que estos pasos también tienen valor, es una actitud que puede favorecer el enfrentarse de forma realista a la investigación y a los artículos sobre elia.

Nuestra intención al poner por escrito estas reflexiones es colaborar en la dirección de mejorar la calidad tanto de las investigaciones como de los articulos en nuestro campo, y que otras personas no tropiecen en los mismos errores que hemos cometido los demás. Esperamos que futuras contribuciones amplíen este debate.

\section{AGRADECIMIENTOS}

Las autoras agradecen a la DGICYT la financiación del proyecto PB94-0629, durante cuya realización tienen su origen estas reflexiones. También a Myriam Krasilchik y Gustav Helldén, un interesante intercambio de ideas; y a Raymond Ricketts, el haber llamado su atencion sobre el trabajo de Wainer.

ESTANY, A. (1993). Introducción a la filosofía de la ciencia. Barcelona: Crítica.

GIERE, R. (1988). Explaining Science. A cognitive approach. Chicago: The University of Chicago Press.

GIL PÉREZ, D. (1994). Diez años de investigación en didáctica de las ciencias: realizaciones y perspectivas. Enseñanza de las Ciencias, 12(2), pp. 154-164.

GILBERT, J. (1994). On the significance of journals in science education: the case of IJSE. International Journal of Science Education, 16(4), pp. 375-384.

HEWSON, P.W. (1981). A conceptual change approach to learning science. European Journal of Science Education, 3(4), pp. 383-396.

HEWSON, P.W., KERBY,H.W. y COOK, P. (1992) Conceptions of Teaching Science held by experienced High School Science Teachers. Paper presented at the NARST meeting, Boston, Massachussets.

HEWSON, P.W. y THORLEY, R. (1989) The conditions of conceptual change in the classroom. International Journal of Science Education, I 1(5), pp. 541-553.

JIMÉNEZ ALEIXANDRE, M.P. (1991). Cambiando las ideas sobre el cambio biológico. Enseñanza de las Ciencias, 9(3), pp. $248-256$. 
JIMÉNEZ ALEIXANDRE, M.P. (1992). Investigación educativa e práctica da aula: métodos cuantitativos e cualitativos. Boletín das Ciencias, 13, pp. 54-58.

JIMÉNEZ, ALEIXANDRE, M.P. (1995). Comments on Editorial Policy Statement and Introduction. Science Education, 79(6), pp. $701-704$.

KELLY, G.J. y CRAWFORD, T. (1996). Looking for Science in the everyday life of a classroom: an analysis of the discoursive processes of school science experimentation. Paper presented at the ann. meeting of NARST, Saint Louis. Abril 1996.

LOPEZ, R., DÍAZ, J., JIMÉNEZ, M.P. y GARCÍA-RODEJA, I. (1992). Educación ambiental: ¿Dentro o fuera de Ja didáctica de las ciencias? Cordoba. IV Encuentro de la Asociación Europea de Didáctica de la Biología.

LUCAS, A.M. (I990). Varieties of Science Education Research: their application in the ciasroom. Enseñanzadelas Ciencias, $8(3), \mathrm{pp} .205-214$

I.UCAS, A.M. y GARCÍA-RODEJA, I. (1990). Contra las interpretaciones simplistas de los experimentos realizados en el aula. Enseñanza de las Ciencias, 8(1), pp. 11-16.

MELLADO, V. y CARRACEDO, D. (1993) Contribuciones de la filosofía de la ciencia a la didáctica đe las ciencias. Enseñanza de las Ciencias, 11(3), pp. 331-339.

MOREIRA, M.A. (1994). Diez años de la revista Enseñanza de las Ciencias: de una ilusión a una realidad. Enseñanza de las Ciencias, 12(2), pp. 147-153.

NOVAK, J. (1982). Teoría y práctica de la educación. Madrid: Alianza.
OSBORNE, R. y WITTROCK, M. (1985). The generative learning model and its implications for teaching. Studies in Science Educution, 12, pp. 59-87.

POSNER, G. J., STRIKE, K., HEWSON, P.W. y GERTZOG, W. (1982). Accommodation of a scientific conception: toward a theory of conceptual change.Science Education, 66(2), pp. 221-227.

RUSSELL, T.L. (1983). Analyzing Arguments in Science classroom discourse: Can teachers' questions distort scientific authority? Journal of Research in Science Teaching, 20(1), pp. $27-45$.

SHERMAN, R. y WEBB, R. (1988) Qualitative Research in Education: Focus and Methods. Hondres: The Fatmer Press.

SHULMAN, L. (1996). A Chat with Lee Shulman. Session 18.49 Annuat Meeting AERA, Nueva York. Abril 1996.

SMITH, E.L., BLAKESLEE, T.D. y ANDERSON,C.W. (1993). Teaching Strategies associated with conceptual change learning in Science. Journal of Research in Science Teaching, 30(2), pp. 11-126.

SOLOMON, J. (1994). The rise and fall of constructivism. Studies in Science Education, 23, pp. 1-19.

THAGARD, P. (1992). Conceptual Revolutions. Princeton, NI: Princeton University Press.

THORLEY, R. (1992). Classroom conceptual ecologies: contrasting discourse in conceptual change instruction. Paper presented at the NARST meeting, Boston, Massachussets.

WAINER, H. (1992). Understanding Graphs and Tables. Educational Researcher, 21(1), pp. 14-23.

[Artículo recibido en marzo de 1995 y aceptado en mayo de 1996.] 
